\title{
Ventilação Oscilatória de Alta Frequência em Neonatologia e Pediatria: uma revisão sistemática
}

\author{
High Frequency Oscillatory Ventilation in Neonatology and Pediatrics: a systematic review
}

\author{
SILVA, Pedro Ykaro Fialho ${ }^{1}$; COSTA, Mayara Fabiana Pereira ${ }^{1}$; BEZERRA, Ingrid Fonsêca \\ Damasceno; ${ }^{1}$ MATIAS, Ana Caryne Xenofonte ${ }^{2}$; PEREIRA, Silvana Alves ${ }^{1,3}$.
}

\begin{abstract}
Resumo
Introdução: A Ventilação Oscilatória de Alta Frequência (VOAF) tem sua eficácia discutida, ao longo dos anos. Existem lacunas na recomendação sobre sua utilização, nas diversas afecções do sistema respiratório, em neonatologia e pediatria. Objetivo: Verificar as principais indicações e repercussões da VOAF, comparadas à ventilação mecânica convencional, nas variáveis respiratórias de recém-nascidos e crianças. Métodos: Foram realizadas, buscas nas bases de dados eletrônicas PubMED e MEDLINE. A seleção dos artigos foi realizada por dois revisores, considerando os ensaios clínicos randomizados, publicados entre os anos de 2014 e 2018, na língua inglesa, com escore PEDro maior que 4, texto completo disponível na íntegra, que abordassem as indicações e repercussões da VOAF em recém-nascidos e crianças. Resultados: Foram incluídos, nove estudos. No que se refere às doenças que resultavam na indicação da VOAF, prevaleceu a síndrome do desconforto respiratório (SDR), tanto na população neonatal quanto na população pediátrica. As variáveis mais estudadas foram as respostas da oxigenação, incluindo índice de oxigenação, pressão parcial de oxigênio, níveis de fração inspirada de oxigênio e níveis de pressão parcial de gás carbônico. Nos estudos que abordaram a VOAF não invasiva, o principal desfecho avaliado foi a necessidade de ventilação mecânica invasiva. Conclusão: A VOAF apresentou resultados superiores, em relação às variáveis de oxigenação, na síndrome do desconforto respiratório moderada a grave, na modalidade invasiva.
\end{abstract}

Palavras-chave: Ventilação de Alta Frequência; Pediatria; Neonatologia; Respiração Artificial.

\footnotetext{
${ }^{1}$ Maternidade Escola Januário Cicco, Universidade Federal do Rio Grande do Norte, UFRN, Natal (RN), Brasil.

${ }^{2}$ Centro Universitário Dr. Leão Sampaio, Juazeiro do Norte (CE), Brasil.

${ }^{3}$ Departamento de Fisioterapia, Universidade Federal do Rio Grande do Norte, UFRN, Natal (RN), Brasil. Email: apsilvana@ccs.ufrn.br
} 


\section{Abstract}

Introduction: High-Frequency Oscillatory Ventilation (HFOV) has its effectiveness discussed over the years. The recommendation of its use in the various respiratory diseases in neonatology and paediatrics is controversial. Objective: To verify the main indications and repercussions of HFOV compared to conventional mechanical ventilation in the respiratory variables of newborns and children. Methods: Searches were performed in the electronic databases PubMed and MEDLINE. The selection of articles was performed by two reviewers, evaluating the randomized clinical trials published between 2014 and 2018, in English, with PEDro score greater than 4, full text available, that addressed HFOV indications and repercussions in newborns and children. Results: 9 studies were included. Regarding the diseases resulted in the indication of HFOV, respiratory distress syndrome (RDS) prevailed, both in the neonatal and pediatric population. The most studied variables were oxygenation responses, including oxygenation index, partial oxygen pressure, levels of inspired oxygen fraction and partial pressure levels of carbon dioxide. In studies addressed non-invasive HFOV, the main outcome assessed was the need for invasive mechanical ventilation. Conclusion: HFOV presented superior results concerning the oxygenation variables in moderate to severe respiratory distress syndrome in invasive modality.

Keywords: High-Frequency Ventilation; Paediatrics; Neonatology; Artificial Respiration.

\section{Introdução}

A ventilação mecânica é uma forma de suporte à vida, amplamente utilizada nas Unidades de Terapia Intensiva (UTIs), tendo, como objetivos, o restabelecimento da função pulmonar, o controle dos distúrbios acidobásicos, a redução do trabalho da musculatura respiratória e a resolução de doenças pulmonares e/ou extrapulmonares ${ }^{1}$.

Apesar de oferecer redução significativa na mortalidade, em pediatria e neonatologia, a ventilação mecânica está relacionada à ocorrência de lesão pulmonar, especialmente, quando elevados parâmetros ventilatórios são utilizados. Para minimizar os riscos associados ao suporte ventilatório, surgiram estratégias protetoras de ventilação mecânica, que defendem o uso de baixos volumes correntes, baixas concentrações de oxigênio e baixos picos de pressão inspiratória ${ }^{2,3}$. Dentre as estratégias de ventilação protetora, em pediatria e neonatologia, a Ventilação Oscilatória de Alta Frequência (VOAF) tem sido descrita como um modo ventilatório capaz de oferecer ventilação e oxigenação adequadas, através de altas frequências oscilatórias no sistema respiratório, prevenindo a hiperdistensão e o colapso de unidades alveolares ${ }^{4,5,6}$.

A VOAF oferece baixos volumes correntes ( 1 a $3 \mathrm{~mL} / \mathrm{kg}$ ) ao sistema respiratório, com uma menor variação de pressão nas vias aéreas e frequências elevadas ( 3 a $50 \mathrm{~Hz}$ ), que podem chegar a 3 mil ciclos

por minuto ${ }^{4,5}$. O uso de altas frequências oscilatórias e de volumes correntes, em níveis próximos ao espaço morto anatômico, resulta em menor grau de lesão pulmonar6 .

Apesar de largamente utilizada, ainda, é necessário apresentar evidências científicas a respeito da VOAF e da sua aplicabilidade. Aparentemente, os resultados são conflitantes, em decorrência do tempo de instalação, das indicações e das repercussões sobre as variáveis respiratórias, nas populações pediátrica e neonatal 7 .

Este estudo tem o objetivo de verificar as principais indicações para o uso da ventilação oscilatória de alta frequência, assim como as repercussões dessa modalidade sobre o sistema respiratório de recémnascidos e crianças, quando comparada à ventilação mecânica convencional. 


\section{Métodos}

Trata-se de uma revisão sistemática baseada na recomendação da Preferred Reporting Items for Systematic reviews and Meta-Analyses (PRISMA) ${ }^{8}$. Para a avaliação da qualidade metodológica dos ensaios clínicos incluídos neste estudo, foi utilizado o escore da Physiotherapy Evidence Database (PEDro) $)^{9}$.

Foi realizada pesquisa retrospectiva de ensaios clínicos randomizados, nas bases de dados PubMED e MEDLINE. Estudos relevantes foram pesquisados, através dos Descritores em Ciências da Saúde (DeCS) "High-Frequency Ventilation", "Neonates" e "Pediatrics". Os operadores booleanos utilizados para a combinação dos descritores e estratégia de pesquisa foram AND e OR. Os artigos foram, inicialmente, extraídos pelo primeiro autor, e as fases subsequentes da pesquisa (avaliação da qualidade metodológica, aplicação dos critérios de elegibilidade e análise dos dados) foram realizadas por pesquisadores independentes. Ensaios clínicos randomizados, publicados entre os anos de $2014 \mathrm{e}$ 2018, foram incluídos nesta revisão.

Estudos que relataram desfechos, em relação à utilização da VOAF, foram incluídos, se (1) a metodologia utilizada fosse caracterizada como ensaio clínico e os indivíduos fossem adequadamente randomizados; (2) os critérios metodológicos apresentassem um escore maior que 4, na Escala PEDro; (3) a terapêutica fosse aplicada nas populações pediátrica e neonatal e os critérios de inclusão estivessem bem estabelecidos, no que diz respeito à faixa etária, tempo de utilização da modalidade ventilatória e (4) apresentassem disponibilidade para leitura na íntegra e fossem escritos na língua inglesa.

\section{Resultados}

Dos 307 ensaios clínicos randomizados identificados nas bases de dados, nove $(n=9)$ cumpriram os critérios e foram incluídos para a composição desta revisão. Fluxograma descrevendo as fases da pesquisa está representado na Figura 1.

No que se refere à aplicabilidade da VOAF, a maior parte dos estudos incluídos discorre sobre a comparação dessa modalidade ventilatória à ventilação mecânica convencional ${ }^{11,12,13,15,16}$. Os principais desfechos utilizados foram a ocorrência de displasia broncopulmonar, retinopatia da prematuridade, hemorragia peri-intraventricular, variáveis gasométricas, tempo de ventilação mecânica invasiva, mortalidade e variáveis de oxigenação, em diversas condições clínicas.

No que se refere às doenças que resultavam na indicação da VOAF, prevaleceu a síndrome do desconforto respiratório (SDR), tanto na população neonatal quanto na população pediátrica ${ }^{11,13,15,17,18}$.

Alguns estudos comparam VOAF não invasiva a outros modos de ventilação não invasiva (VNI). A utilização da VOAF foi indicada, especialmente, nos casos de falha de outros modos de VNI. Os principais desfechos avaliados nestas condições foram a necessidade de intubação orotraqueal, níveis de $\mathrm{PaCO}_{2}$, eventos de apneia e/ou pausa respiratória, além de níveis de oxigenação $0^{18,20,21}$.

A Tabela 1 apresenta os dados extraídos dos ensaios clínicos incluídos, com suas principais características. 
Figura 1 | Fases propostas pela recomendação PRISMA.

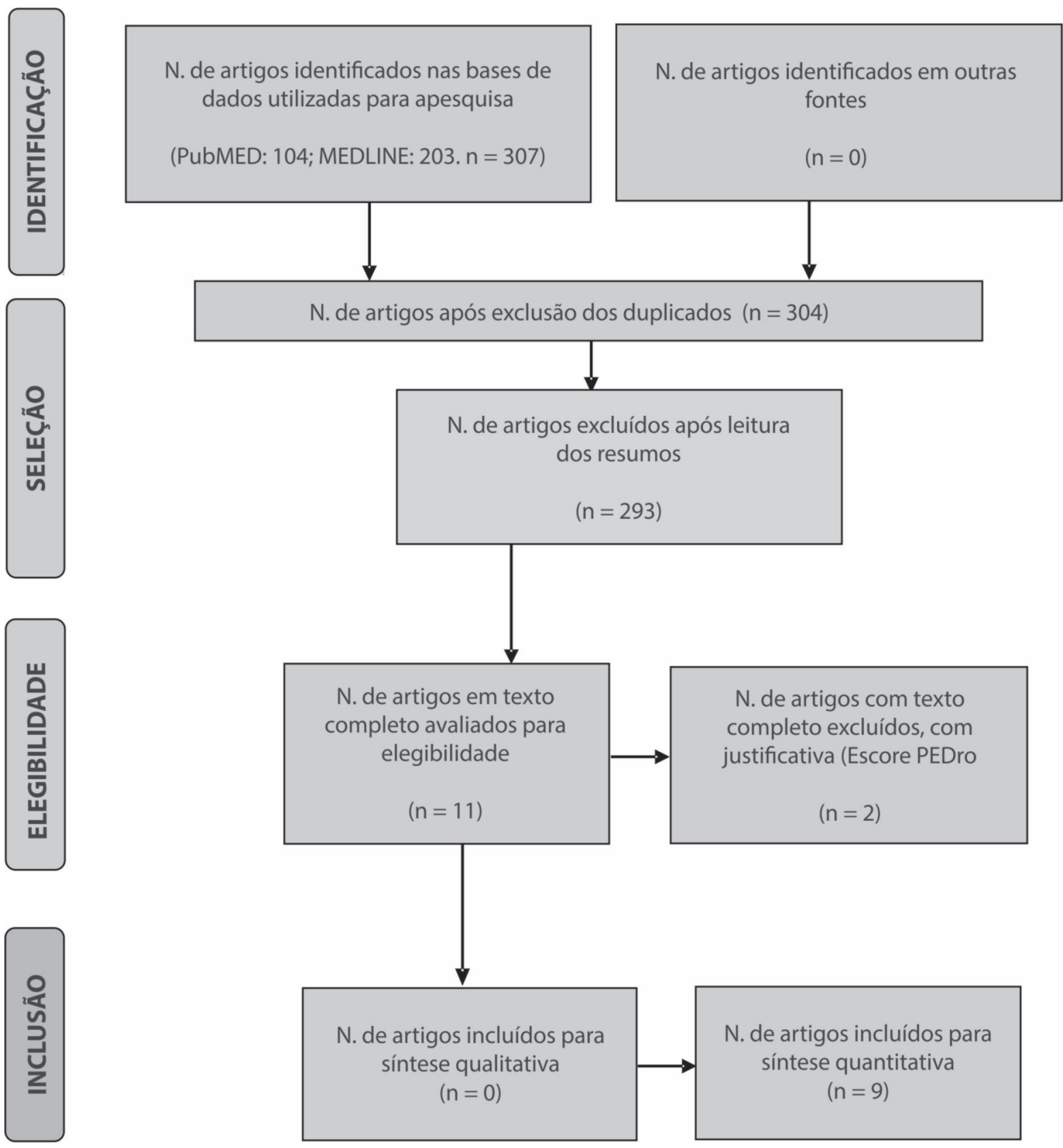

Fonte: Adaptado de Moher et al. (2009) 
Tabela 1 | Síntese dos artigos incluídos para a revisão sistemática.

\begin{tabular}{|c|c|c|c|c|c|c|}
\hline $\begin{array}{l}\text { Autor } \\
\text { (Ano) }\end{array}$ & Objetivo & $\mathbf{n}$ & Características & Intervenção & Resultados & $\begin{array}{l}\text { Escore } \\
\text { PEDro }\end{array}$ \\
\hline $\begin{array}{l}\text { Iscan et al. } \\
\text { (2015) }\end{array}$ & $\begin{array}{l}\text { Investigar se a } \\
\text { VOAF com VG } \\
\text { pode resultar em } \\
\text { menor variação } \\
\text { dos níveis de } \\
\mathrm{PCO}_{2} \text {, em } \\
\text { comparação à }_{\text {VOAF sem VG }}\end{array}$ & 20 & $\begin{array}{l}\text { RNs com média de } \\
\text { idade gestacional de } \\
28 \pm 2.4 \text { semanas e } \\
\text { média de peso de } \\
1.080 \mathrm{~g} \pm 390 \mathrm{~g}\end{array}$ & $\begin{array}{l}\text { VOAF com VG } \\
\text { versus VOAF } \\
\text { sem VG }\end{array}$ & $\begin{array}{l}\text { VOAF com VG } \\
\text { oferece uma } \\
\text { maior } \\
\text { estabilidade do } \\
\text { VC. Incidência de } \\
\text { hipocarbia e } \\
\text { hipercarbia foi } \\
\text { maior no grupo } \\
\text { de VOAF sem VG }\end{array}$ & $7 / 10$ \\
\hline $\begin{array}{l}\text { Klotz et al. } \\
\text { (2017) }\end{array}$ & $\begin{array}{l}\text { Determinar se a } \\
\text { VOAF não } \\
\text { invasiva } \\
\text { é mais eficaz na } \\
\text { redução dos } \\
\text { níveis de } \mathrm{PCO}_{2} \text {, } \\
\text { em comparação } \\
\text { ao CPAP }\end{array}$ & 26 & $\begin{array}{c}\text { Média de peso de } \\
819.2 \mathrm{~g} \pm 208.2 \mathrm{~g} \text { na } \\
\text { sequência VOAF- } \\
\text { CPAP e de } 1067.3 \pm \\
332.9 \text { na sequência } \\
\text { CPAP-VOAF; média } \\
\text { de idade gestacional } \\
\text { de } 26.1 \pm 2.2 \\
\text { semanas na primeira } \\
\text { sequência e de } 27.2 \pm \\
2.0 \text { semanas na } \\
\text { segunda sequência } \\
\text { de tratamento }\end{array}$ & $\begin{array}{c}\text { VOAF versus } \\
\text { CPAP }\end{array}$ & $\begin{array}{c}\text { Não houve } \\
\text { diferenças } \\
\text { significativas em } \\
\text { todas as variáveis } \\
\text { estudadas }\end{array}$ & $7 / 10$ \\
\hline $\begin{array}{l}\text { Zivanovic } \\
\text { et al. } \\
(2014)\end{array}$ & $\begin{array}{l}\text { Analisar as } \\
\text { repercussões da } \\
\text { VOAF e da VMI } \\
\text { convencional nas } \\
\text { variáveis de } \\
\text { função pulmonar, } \\
\text { qualidade de } \\
\text { vida e estado } \\
\text { funcional em } \\
\text { crianças que } \\
\text { necessitaram de } \\
\text { VMI ao } \\
\text { nascimento }\end{array}$ & 797 & $\begin{array}{c}\text { Média de idade } \\
\text { gestacional de } 27.0 \pm \\
1.2 \text { semanas no } \\
\text { grupo de ventilação } \\
\text { convencional e de } \\
26.7 \pm 1.5 \text { semanas } \\
\text { no grupo VOAF; } \\
\text { Média de peso de } \\
923 \mathrm{~g} \pm 206 \mathrm{~g} \text { no } \\
\text { grupo de ventilação } \\
\text { convencional e } 867 \mathrm{~g} \\
\pm 209 \mathrm{~g} \text { no grupo de } \\
\text { VOAF }\end{array}$ & $\begin{array}{l}\text { VOAF versus } \\
\text { VMI } \\
\text { convencional }\end{array}$ & $\begin{array}{l}\text { O grupo que } \\
\text { recebeu a VOAF } \\
\text { apresentou } \\
\text { resultados } \\
\text { superiores em } \\
\text { testes de função } \\
\text { pulmonar, com } \\
\text { melhores níveis } \\
\text { de VEF1, CVF e } \\
\text { FEF }_{75 \%}\end{array}$ & $5 / 10$ \\
\hline $\begin{array}{l}\text { Zhu et al. } \\
\text { (2017) }\end{array}$ & $\begin{array}{c}\text { Comparar o } \\
\text { efeito da VOAF } \\
\text { não invasiva ao } \\
\text { CPAP em } \\
\text { prematuros com } \\
\text { SDR, após técnica } \\
\text { INSURE, } \\
\text { analisando a } \\
\text { necessidade de } \\
\text { VMI nos dois } \\
\text { modos de } \\
\text { ventilação }\end{array}$ & 76 & $\begin{array}{c}\text { Média de } 31.7 \pm 1.7 \\
\text { semanas no grupo } \\
\text { VOAF e } 32.0 \pm 1.9 \text { no } \\
\text { grupo CPAP; Média } \\
\text { de peso de } 1670 \mathrm{~g} \pm \\
353 \mathrm{~g} \text { no grupo VOAF } \\
\text { e de } 1735 \mathrm{~g} \pm 327 \mathrm{~g} \text { no } \\
\text { grupo CPAP }\end{array}$ & $\begin{array}{c}\text { VOAF não } \\
\text { invasiva versus } \\
\text { CPAP }\end{array}$ & $\begin{array}{l}\text { A necessidade de } \\
\text { VMI foi menor no } \\
\text { grupo VOAF em } \\
\text { comparação ao } \\
\text { grupo CPAP. A } \\
\text { incidência de } \\
\text { hemorragia } \\
\text { intraventricular, } \\
\text { escapes de ar ou } \\
\text { DBP foi } \\
\text { semelhante entre } \\
\text { os grupos }\end{array}$ & $6 / 10$ \\
\hline
\end{tabular}


Tabela 1 | Síntese dos artigos incluídos para a revisão sistemática.

\begin{tabular}{|c|c|c|c|c|c|c|}
\hline $\begin{array}{l}\text { Autor } \\
\text { (Ano) }\end{array}$ & Objetivo & $\mathbf{n}$ & Características & Intervenção & Resultados & $\begin{array}{l}\text { Escore } \\
\text { PEDro }\end{array}$ \\
\hline $\begin{array}{l}\text { Zhou et al. } \\
\text { (2017) }\end{array}$ & $\begin{array}{c}\text { Comparar a } \\
\text { eficácia de } \\
\text { diferentes modos } \\
\text { ventilatórios } \\
\text { associados à } \\
\text { terapia } \\
\text { secretolítica com } \\
\text { ambroxol em } \\
\text { prematuros com } \\
\text { SDR }\end{array}$ & 73 & $\begin{array}{c}\text { Média de idade } \\
\text { gestacional de } 32.35 \\
\pm 1.95 \text { semanas no } \\
\text { grupo VMI e média } \\
\text { de } 33.13 \pm 2.04 \\
\text { semanas no grupo } \\
\text { VOAF }\end{array}$ & $\begin{array}{l}\text { VOAF versus } \\
\text { VMI } \\
\text { convencional, } \\
\text { ambos } \\
\text { associados à } \\
\text { terapia } \\
\text { secretolítica }\end{array}$ & $\begin{array}{c}\text { Melhores } \\
\text { resultados em } \\
\text { relação aos } \\
\text { parâmetros de } \\
\text { oxigenação } \\
\left(\mathrm{PaO}_{2}, \mathrm{IO} \text { e }\right. \\
\text { relação } \\
\left.\mathrm{PaO}_{2} / \mathrm{PAO}_{2}\right) \text { no } \\
\text { grupo VOAF. Sem } \\
\text { diferença } \\
\text { significativa entre } \\
\text { os grupos em } \\
\text { relação à } \\
\text { mortalidade, } \\
\text { pneumotórax, } \\
\text { DBP, retinopatia } \\
\text { da } \\
\text { prematuridade, } \\
\text { hemorragia } \\
\text { intraventricular, } \\
\text { leucomalácia } \\
\text { periventricular e } \\
\text { tempo de VMI }\end{array}$ & $7 / 10$ \\
\hline $\begin{array}{l}\text { Snoek et } \\
\text { al. (2016) }\end{array}$ & $\begin{array}{l}\text { Determinar qual } \\
\text { a modalidade } \\
\text { ventilatória ideal } \\
\text { para pacientes } \\
\text { com hérnia } \\
\text { diafragmática, } \\
\text { através da } \\
\text { comparação } \\
\text { entre VOAF e VMI } \\
\text { convencional }\end{array}$ & 171 & $\begin{array}{c}\text { Média de idade } \\
\text { gestacional de } 38 \\
(37.3 \text { - 39.0) semanas } \\
\text { no grupo VOAF e } \\
\text { média de } 38.1 \text { ( } 37.4 \text { - } \\
\text { 38.9) semanas no } \\
\text { grupo VMI } \\
\text { convencional }\end{array}$ & $\begin{array}{c}\text { VOAF versus } \\
\text { VMI } \\
\text { convencional }\end{array}$ & $\begin{array}{l}\text { O estudo não } \\
\text { demonstrou } \\
\text { diferenças } \\
\text { estatisticamente } \\
\text { significativas nos } \\
\text { índices de } \\
\text { mortalidade, DBP } \\
\text { e tempo de VMI }\end{array}$ & $7 / 10$ \\
\hline $\begin{array}{l}\text { Samransa } \\
\text { mruajkit } \\
\text { et al. } \\
(2018)\end{array}$ & $\begin{array}{l}\text { Determinar os } \\
\text { efeitos da } \\
\text { manobra de } \\
\text { recrutamento } \\
\text { alveolar com } \\
\text { VOAF e com a } \\
\text { VMI convencional } \\
\text { nas variáveis } \\
\text { hemodinâmicas e } \\
\text { de oxigenação, } \\
\text { assim como os } \\
\text { desfechos } \\
\text { clínicos desta } \\
\text { intervenção em } \\
\text { crianças com SDR } \\
\text { pediátrica }\end{array}$ & 18 & $\begin{array}{c}\text { Média de idade de } \\
47.7 \pm 61.2 \text { meses; } \\
\text { média de peso de } \\
25.3 \pm 27.1 \\
\text { quilogramas }\end{array}$ & $\begin{array}{l}\text { Manobra de } \\
\text { recrutamento } \\
\text { alveolar em } \\
\text { VOAF e em VMI } \\
\text { convencional }\end{array}$ & $\begin{array}{l}\text { VOAF combinada } \\
\text { com manobra de } \\
\text { recrutamento } \\
\text { apresentou } \\
\text { melhores } \\
\text { resultados nas } \\
\text { variáveis de } \\
\text { oxigenação, } \\
\text { assim como } \\
\text { melhor efeito } \\
\text { clínico em } \\
\text { comparação à } \\
\text { VMI convencional }\end{array}$ & $6 / 10$ \\
\hline
\end{tabular}


Tabela 1 | Síntese dos artigos incluídos para a revisão sistemática.

\begin{tabular}{|c|c|c|c|c|c|c|}
\hline $\begin{array}{l}\text { Autor } \\
\text { (Ano) }\end{array}$ & Objetivo & $n$ & Características & Intervenção & Resultados & $\begin{array}{l}\text { Escore } \\
\text { PEDro }\end{array}$ \\
\hline $\begin{array}{c}\text { El- } \\
\text { Nawawy } \\
\text { et al. } \\
(2017)\end{array}$ & $\begin{array}{l}\text { Comparar os } \\
\text { resultados do uso } \\
\text { precoce de VOAF } \\
\text { ou VMI } \\
\text { convencional em } \\
\text { pacientes com } \\
\text { SDR pediátrica }\end{array}$ & 200 & $\begin{array}{l}\text { Média de idade de } \\
7.75 \text { meses no grupo } \\
\text { VMI convencional e } \\
\text { de } 7.0 \text { meses no } \\
\text { grupo VOAF; Média } \\
\text { de peso de } 7.8 \\
\text { quilogramas no } \\
\text { grupo VMI } \\
\text { convencional e } 8.0 \\
\text { quilogramas no } \\
\text { grupo VOAF }\end{array}$ & $\begin{array}{c}\text { VOAF versus } \\
\text { VMI } \\
\text { convencional }\end{array}$ & $\begin{array}{l}\text { O grupo VOAF } \\
\text { apresentou } \\
\text { melhora } \\
\text { significativa das } \\
\text { variáveis de } \\
\text { oxigenação após } \\
24 \text { horas de } \\
\text { utilização. Não } \\
\text { foram } \\
\text { encontrados } \\
\text { resultados } \\
\text { estatisticamente } \\
\text { significativos nas } \\
\text { taxas de } \\
\text { mortalidade e } \\
\text { tempo de VMI } \\
\text { entre os grupos }\end{array}$ & $5 / 10$ \\
\hline
\end{tabular}

Fonte: Dados da pesquisa (2020). n: número de indivíduos na amostra do estudo. RNs: Recém-nascidos. VG: Volume Garantido. VOAF: Ventilação Oscilatória de Alta Frequência. SDR: Síndrome do desconforto respiratório. $V_{E F}$ : Volume Expiratório Forçado no Primeiro Segundo. CVF: Capacidade Vital Forçada. BiPAP: BI-level Positive Airway Pressure. VC: Volume Corrente. $\mathrm{PCO}_{2}$ : Pressão Parcial de Gás Carbônico. DBP: Displasia Broncopulmonar VNI: Ventilação Não Invasiva. VMI: Ventilação Mecânica Invasiva. IO: Índice de Oxigenação. FiO ${ }_{2}$ : Fração Inspirada de Oxigênio. CPAP: Continuous Positive Airway Pressure. INSURE: Intubação-Surfactante-Extubação. $\mathrm{FEF}_{75 \%}$ : Fluxo Expiratório Forçado em $75 \%$.

\section{Discussão}

A presente revisão selecionou nove ensaios clínicos randomizados, que descrevem a utilização da VOAF, em comparação à ventilação mecânica convencional, seja de forma invasiva ou não invasiva, e avaliou as repercussões respiratórias desses modos ventilatórios, em pediatria e neonatologia.

Em estudos que indicaram a VOAF, em comparação com a ventilação mecânica convencional, em recém-nascidos prematuros diagnosticados com SDR, os principais desfechos analisadas foram a oxigenação $\left(\mathrm{PaO}_{2}\right.$, níveis de $\mathrm{FiO}_{2}$, índice de oxigenação, relação $\mathrm{PaO}_{2} / \mathrm{FiO}_{2}$, e gradiente alvéolo-arterial de oxigênio), níveis de $\mathrm{PaCO}_{2}$ e complicações como pneumotórax, retinopatia da prematuridade, displasia broncopulmonar e mortalidade ${ }^{11}$. Há descrição da melhora significativa, nas variáveis de oxigenação, no grupo VOAF, em comparação à ventilação mecânica convencional, exemplificado pelo aumento dos níveis de $\mathrm{PaO}_{2}$, menor necessidade de altos níveis de $\mathrm{FiO}_{2}$ e melhores índices de oxigenação, após 24 horas. Os níveis de $\mathrm{PaCO}_{2}$, também, foram mais bem controlados na VOAF. Não houve diferença significativa, em relação às outras variáveis analisadas ${ }^{11}$.

Nota-se que os resultados, quanto às variáveis de oxigenação, podem estar relacionadas ao mecanismo de manutenção do volume pulmonar oferecido pela VOAF. A utilização de baixos níveis de volume corrente (VC) e a manutenção de pressão média de vias aéreas acima da pressão de fechamento alveolar podem ser úteis para a manutenção do volume pulmonar e oferecem efeito protetor, ao evitar 
hiperdistensão e colapso alveolar. A necessidade de níveis mais baixos de $\mathrm{FiO}_{2}$ nessa modalidade, encontrada em alguns estudos ${ }^{10,11}$, também, oferece maior proteção aos pulmões, em virtude da menor exposição à toxicidade do oxigênio.

O mecanismo fisiológico da utilização da VOAF pode dar subsídio ao entendimento das variáveis de oxigenação dessa modalidade de ventilação. $O$ controle da pressão média, nas vias aéreas, mantém a abertura das unidades alveolares e a oxigenação do sistema respiratório ${ }^{14}$.

Em crianças com diagnóstico de SDR moderada a grave, a VOAF tem apresentado superioridade, quando comparada à ventilação mecânica convencional, no que diz respeito às variáveis de oxigenação, com maior valor $\mathrm{de} \mathrm{PaO}_{2} / \mathrm{FiO}_{2}$, redução do índice de oxigenação e menor necessidade de $\mathrm{FiO}_{2}$. Apesar disso, não há diferença significativa, entre os dois modos na mortalidade e tempo de ventilação mecânica ${ }^{13,15}$.

A VOAF, também, é indicada em pacientes com hérnia diafragmática congênital ${ }^{2}$. Ao compará-la com a ventilação mecânica convencional, não houve diferença significativa, entre os dois modos ventilatórios, nas taxas de mortalidade e displasia broncopulmonar. Entretanto, os recém-nascidos ventilados com VOAF apresentaram maior ocorrência de hipertensão pulmonar e maior tempo em ventilação mecânica ${ }^{12}$.

Indicações, também, utilizadas pelos estudos para VOAF foram as cardiopatias sem grande repercussão hemodinâmica, a presença de relação $\mathrm{PaO}_{2} / \mathrm{FiO}_{2}$ menor ou igual a $200 \mathrm{mmHg}$ e a presença de infiltrados pulmonares bilaterais na radiografia de tórax. Outros achados, que caracterizassem a SDR moderada a grave, também, foram considerados ${ }^{13,15,17,18}$.

Outra possibilidade de utilização da VOAF é sua associação com a modalidade volume garantido. A escolha de volume alvo, a ser atingido em cada ciclo respiratório, foi capaz de promover melhor ventilação e troca gasosa mais eficaz, em comparação à utilização da VOAF, sem a garantia de volume. A diferença entre os dois modos se dá, principalmente, pela provável regulação dos níveis de $\mathrm{PaCO}_{2}$ no primeiro modo, uma vez que o $\mathrm{VC}$ se torna variável de controle ${ }^{17}$.

O tempo de indicação da VOAF permanece controverso, especialmente, pela sua descrição inicial, como modo de resgate à ventilação mecânica convencional. Apesar disso, novos estudos têm demonstrado resultados favoráveis, no que se refere à efetividade da VOAF como modalidade de primeira escolha. O uso precoce da VOAF, em casos de SDR pediátrica, foi capaz de gerar um decréscimo significativo no índice de oxigenação, com melhora considerável da hipoxemia, nas primeiras 24 horas de utilização. Apesar disso, não houve diferenças entre os grupos, em relação ao tempo de permanência na UTI e ao tempo de internação hospitalar, de uma forma geral ${ }^{15,17}$.

O principal achado da aplicação da VOAF, como medida ventilatória inicial, é a melhora das trocas gasosas e, consequentemente, da oxigenação. Não foram relatados efeitos adversos nas variáveis hemodinâmicas, quando esta estratégia foi comparada à ventilação mecânica convencional. Além disso, não houve associação entre esta estratégia e a ocorrência de eventos, que pudessem predispor a uma maior taxa de mortalidade ou morbidade nas populações estudadas, assim como no desenvolvimento de displasia broncopulmonar ${ }^{11,12,13}$.

Associada ao uso da VOAF, a manobra de recrutamento alveolar, executada de forma prévia à sua instalação, tem demonstrado melhora significativa nas variáveis de oxigenação ${ }^{13}$. Houve redução importante do índice de oxigenação dos pacientes com SDR, que receberam o recrutamento alveolar no modo VOAF, em comparação ao modo de ventilação convencional. A manobra de recrutamento, no modo de ventilação mecânica convencional, apresentou ineficiência na redução da $\mathrm{FiO}_{2}{ }^{13}$.

A VOAF, também, pode ser utilizada de forma não invasiva ${ }^{19}$. O uso da VOAF não invasiva, em comparação ao modo CPAP, em crianças prematuras com SDR moderada a grave, demonstrou 
menor necessidade de ventilação mecânica invasiva no grupo que recebeu a VOAF, como descrito em um estudo ${ }^{18}$. Os critérios que constituíram a falha de ventilação mecânica não invasiva envolveram a presença de acidose respiratória, hipóxia, hemorragia pulmonar, apneia e outras variáveis clínicas, que foram mais comuns no modo $\mathrm{CPAP}^{18}$. Em outros estudos, não foram observadas diferenças entre a VOAF não invasiva e ventilação não invasiva (VNI - dois níveis pressóricos) em crianças prematuras. Os níveis de $\mathrm{PaCO}_{2}$ foram similares nos dois grupos, sem superioridade de um modo de VNI sobre o outro ${ }^{20,21}$. Cabe ressaltar que o uso de diferentes interfaces para a VNI pode ter grande influência nos resultados encontrados. Adicionalmente, o escape de ar, durante a aplicação da VNI, é fator que favorece a falha da estratégia e a maior variação nos níveis de $\mathrm{PaCO}_{2}$.

\section{Conclusão}

De acordo com os estudos apresentados, as principais indicações da VOAF foram a SDR moderada a grave e a prematuridade extrema. Nos estudos de comparação entre a VOAF e a ventilação mecânica convencional, a VOAF parece apresentar resultados superiores, em relação às variáveis de oxigenação, e não houve diferença, estatisticamente significativa, na ocorrência de complicações.

Pacientes com necessidade de ventilação mecânica, devidoà hérnia diafragmática congênita, também, tiveram indicação da VOAF, mas, sem diferença, ao comparar com ventilação mecânica convencional.

Em relação à VOAF não invasiva, esta apresentou discreta vantagem, em relação ao CPAP.

\section{Referências}

1.CarvalhoCR, ToufenJuniorC,FrancaSA. Ventilaçãomecânica:princípios, análisegráficaemodalidades ventilatórias. J Bras Pneumol. 2007 Jul;33(Supl 2):54-70. doi: 10.1590/S1806-37132007000800002.

2. Sutherasan Y, Vargas M, Pelosi P. Protective mechanical ventilation in the non-injured lung: review and meta-analysis. Crit Care. 2014 Mar 18;18(2):211. doi: 10.1186/cc13778.

3. Seiberlich E, Santana JA, Chaves RA, Seiberlich RC. [Protective Mechanical Ventilation, why use it?] Rev Bras Anestesiol. 2011 Sep-Oct;61(5):659-67, 361-5. doi: 10.1016/S0034-7094(11)70076-9.

4. Cools F, Offringa M, Askie LM. Elective high frequency oscillatory ventilation versus conventional ventilation for acute pulmonary dysfunction in preterm infants. Cochrane Database Syst Rev. 2015 Mar 19;(3):CD000104. doi: 10.1002/14651858.CD000104.pub4.

5. Pinto JR, Diniz EMA, Vaz FAC. Ventilação de alta frequência oscilatória em recém-nascidos prematuros. Pediatria. 2004;26:188-197.

6. Henderson-Smart DJ, Bhuta T, Cools F, Offringa M. Elective high frequency oscillatory ventilation versus conventional ventilation for acute pulmonary dysfunction in preterm infants. Cochrane Database Syst Rev. 2007 Jul 18;(3):CD000104. doi: 10.1002/14651858.CD000104.pub2.

7. Rimensberger PC, Beghett M, Hanquinett S, Berner M. First intention high-frequency oscillation with early lung volume optimization improves pulmonary outcome in very low birth weight infants with respiratory distress syndrome. Pediatrics. 2000 Jun;105(6):1202-8. doi: 10.1542/peds.105.6.1202.

8. Moher D, Liberati A, Tetzlaff J, Altman DG. Preferred reporting items for systematic reviews and meta-analyses: the PRISMA statement. Ann Intern Med. 2009 Aug 18;151(4):264-9, W64. doi: 10.7326/0003-4819-151-4-200908180-00135.

9. Verhagen AP, De Vet HC, De Bie RA, Kessels AG, Boers M, Bouter LM, et al. The Delphi list: a criteria 
list for quality assessment of randomized clinical trials for conducting systematic reviews developed by Delphi consensus. J Clin Epidemiol. 1998 Dec;51(12):1235-41. doi: 10.1016/s0895-4356(98)00131-0.

10. Cummings JJ, Polin RA. Noninvasive Respiratory Support. Pediatrics. 2016 Jan;137(1). doi: 10.1542/ peds.2015-3758. Epub 2015 Dec 29.

11. Zhou B, Zhai JF, Wu JB, Jin B, Zhang YY. Different Ventilation Modes Combined With Ambroxol In The Treatment Of Respiratory Distress Syndrome In Premature Infants. Exp Ther Med. 2017 Feb;13(2):629-633. doi: 10.3892/etm.2016.3978. Epub 2016 Dec 16.

12. Snoek KG, Capolupo I, van Rosmalen J, Hout LJ, VijfhuizeS, Greenough A, et al. Conventional mechanical ventilation versus high-frequency oscillatory ventilation for congenital diaphragmatic hernia: a randomized clinical trial (the VICI-trial). Ann Surg. 2016 May;263(5):867-74. doi: 10.1097/SLA.0000000000001533.

13. Samransamruajkit R, Rassameehirun C, Pongsanon K, Huntrakul S, Deerojanawong J, Sritippayawan $S$, et al. A comparison of clinical efficacy between high frequency oscillatory ventilation and conventional ventilation with lung volume recruitment in pediatric acute respiratory distress syndrome: A randomized controlled trial. Indian J Crit Care Med. 2016 Feb;20(2):72-7. doi: 10.4103/0972-5229.175940.

14. Fioretto JR, Oliveira MA, Rebello CM. Ventilación de alta frecuencia em em recién nacido y em el niño. In: Carvalho WB, Jiménes HJ, Sásbon JS. Ventilación pulmonar mecânica em pediatria. São Paulo: Atheneu; 2012. p. 337-56.

15. El-Nawawy A, Moustafa A, Heshmat H, Abouahmed A. High frequency oscillatory ventilation versus conventional mechanical ventilation in pediatric acute respiratory distress syndrome: a randomized controlled study. Turk J Pediatr. 2017;59(2):130-143. doi: 10.24953/turkjped.2017.02.004.

16. Zivanovic S, Peacock J, Alcazar-Paris M, Lo JW, Lunt A, Marlow N, et al. Late outcomes of a randomized trial of high-frequency oscillation in neonates. N Engl J Med. 2014 Mar 20;370(12):11211130. doi: 10.1056/NEJMoa1309220.

17. Iscan B, Duman N, Tuzun F, Kumral A, Ozkan H. Impact of volume guarantee in high-frequency oscillatory ventilation in preterm infants: a randomized crossover clinical trial. Neonatology. 2015;108(4):277-82. doi: 10.1159/000437204. Epub 2015 Sep 1.

18. Zhu X-W, Zhao J-N, Tang S-F, Yan J, Shi Y. Noninvasive high-frequency oscillatory ventilation versus nasal continuous positive airway pressure in preterm infants with moderate-severe respiratory distress syndrome: a preliminary report. Pediatr Pulmonol. 2017 Aug;52(8):1038-1042. doi: 10.1002/ ppul.23755. Epub 2017 Jul 3.

19. Fischer HS, Bohlin K, Bührer C, Schmalisch G, Cremer M, Reiss I, et al. Nasal high-frequency oscillation ventilation in neonates: a survey in five European countries. Eur J Pediatr. 2015 Apr;174(4):46571. doi: 10.1007/s00431-014-2419-y. Epub 2014 Sep 18.

20. Klotz D, Schneider H, Schumann S, Mayer B, Fuchs H. Noninvasive high-frequency oscillatory ventilation in preterm infants: a randomised controlled crossover trial. Arch Dis Child Fetal Neonatal Ed. 2018 Jul;103(4):F1-F5. doi: 10.1136/archdischild-2017-313190. Epub 2017 Sep 16.

21. Mukerji A, Sarmiento K, Lee B, Hassall K, Shah V. Non-invasive high-frequency ventilation versus bi-phasic continuous positive airway pressure (BP-CPAP) following CPAP failure in infants <1250 g: a pilot randomized controlled trial. J Perinatol. 2017 Jan;37(1):49-53. doi: 10.1038/jp.2016.172. Epub 2016 Sep 29.

Submissão em: 10/10/2019

Aceito em: 13/07/2020 